\title{
A investigação do impacto do autismo na família: Revisão crítica da literatura e proposta de um novo modelo
}

\author{
Carlo Schmidt \\ Cleonice Bosa \\ Universidade Federal do Rio Grande do Sul
}

\begin{abstract}
Resumo
Os Transtornos Globais do Desenvolvimento, dos quais o autismo faz parte, caracterizam-se pelo comprometimento severo em três áreas do desenvolvimento: habilidades de interação social recíproca, habilidades de comunicação e presença de comportamentos, interesses e atividades estereotipadas (DSM-IV-TR, 2002). As características próprias do comportamento, somadas à severidade do transtorno podem constituir estressores em potencial para familiares e/ou cuidadores. O presente estudo tem como objetivo revisar as pesquisas sobre o impacto dos Transtornos Globais do Desenvolvimento, em especial do autismo, na família. A partir da noção de que este fenômeno envolve uma série de fatores interatuantes, intra e extrafamiliares os quais afetam a família ao longo de seu ciclo vital, conclui-se que a sua compreensão não pode ocorrer com base em relações lineares entre possíveis causas e seus efeitos, de forma reducionista. Como alternativa, o estudo aponta para a necessidade da adoção de um modelo explicativo que contemple as diversas variáveis envolvidas no processo de adaptação da família frente a uma condição crônica. Destaca-se o modelo de Bradford (1997), o qual propõe uma abordagem psicossocial, que integra concepções cognitivas e sistêmicas, na área da Psicologia da Saúde, num metamodelo especificamente desenvolvido para o estudo do impacto da doença crônica na família.
\end{abstract}

Palavras-chave: Autismo; estresse familiar; impacto na família.

\begin{abstract}
The autism burden in the family: A critical review and a new model proposal

Pervasive developmental disorders, including autism, consist of a severe impairment in three developmental areas: impairment of social skills; impairment of communication; and stereotypical behaviors, interests and activities (DSM-IV-TR, 2002). The behavior characteristics associated with the disorder severity may be potential stressors for family, relatives and caregivers. The burden on the family depends upon a range of interacting factors which require an explaining model which takes into account the variables involved in the family's adaptation to this chronic condition. We argue that the Bradford's biopsychosocial model (1997), although originating in the health psychology field, may be useful for understanding developmental disorders to the extend that it emphasizes interactions between the following contextual factors; health beliefs, social support, disease challenges, coping strategies, and family communication patterns. The purpose of the present study is to make a critical review on studies regarding the impact of Pervasive Developmental Disorders in the family. As this phenomenon involves a range of interacting factors, in and outside the family and affect it's vital cycle, we conclude that understanding could not be based upon linear relations between causes and its outcomes. As an alternative, this paper suggests the need of an explaining model with respect to the range of variables involved on the family adaptative process when facing chronic disease. We emphasize the Bradford's model (1997), which provides a psychosocial framework that integrates cognitive and systemic conceptions in a metamodel, specifically developed for a study of the impact of chronic illness in family.
\end{abstract}

Keywords: Autism; family stress; burden of care.

Os Transtornos Globais do Desenvolvimento (TGDs), os quais incluem o autismo, têm sido definidos como uma síndrome, envolvendo comprometimento severo e invasivo em três áreas do desenvolvimento: habilidades de interação social recíproca, habilidades de comunicação, e presença de comportamentos, interesses e atividades estereotipadas (DSM-IV-TR, 2002). Estas características podem constituir estressores em potencial para familiares e/ou cuidadores. A partir da revisão da literatura, observa-se que estudos internacionais, que tratam do impacto dos TGDs sobre os pais, têm utilizado predominantemente escalas fechadas como instrumento de medida (Ex.: estresse parental), obtendo dados importantes, porém limitados, ao passo que estudos brasileiros nessa área têm sido raros e fundamentados em um "modelo de déficit" em contraste ao de "recursos". Em um modelo de déficit é ressaltada a questão da psicopatologia familiar, enquanto que no de recursos, focalizam-se, além da identificação de comprometimentos, as 
estratégias que as famílias desenvolvem para compreender e lidar com a síndrome.

$\mathrm{O}$ presente estudo tem como objetivo revisar as pesquisas sobre o impacto dos Transtornos Globais do Desenvolvimento, em especial do autismo, na família. A partir da noção de que este fenômeno envolve uma série de fatores interatuantes, intra e extrafamiliares (Ex.: especificidades da síndrome, rede de apoio, crenças familiares sobre a síndrome) e afetam a família ao longo de seu ciclo vital, conclui-se que a sua compreensão não pode ocorrer com base em relações lineares entre possíveis causas e seus efeitos, de forma reducionista. Como alternativa, o estudo aponta para a necessidade da adoção de um modelo explicativo que contemple as diversas variáveis envolvidas no processo de adaptação da família frente a uma condição crônica. Esta particularidade, isto é, o fato do comprometimento do desenvolvimento ter seu início nos primeiros anos de vida da criança e, portanto, se estender por diferentes períodos evolutivos da criança, tem sido negligenciado nas abordagens tradicionais. Destaca-se o modelo de Bradford (1997), o qual propõe uma abordagem psicossocial, que integra concepções cognitivas e sistêmicas, na área da Psicologia da Saúde, num metamodelo especificamente desenvolvido para o estudo do impacto da doença crônica na família.

\section{Autismo}

As primeiras publicações a respeito da síndrome do autismo foram de autoria do psiquiatra austríaco Leo Kanner, em 1943, denominando-a inicialmente de "distúrbio autístico do contato afetivo". Logo depois, esse termo foi substituído por "autismo infantil precoce" (Kanner, 1944). Kanner descreveu o caso de onze crianças que apresentavam um quadro por ele considerado raro, no qual o comprometimento fundamental era a incapacidade para relacionamentos interpessoais, desde o início da vida.

Estas primeiras idéias ainda destacaram como denominador comum aos 11 casos, os altos níveis de inteligência e sociocultural dos pais das crianças avaliadas, bem como obsessividade e certa frieza nas relações entre casais e pais e filhos. No entanto, Kanner logo questionou o papel da psicopatologia parental como possível etiologia do autismo, colocando a dificuldade em se atribuir todo o quadro exclusivamente ao tipo de relações parentais precoces. Sua conclusão foi de que o autismo origina-se de uma incapacidade inata de estabelecer o contato afetivo habitual e biologicamente previsto com as pessoas (Kanner, 1943), sem, contudo, excluir a importância dos aspectos ambientais no desenvolvimento.

O conceito de autismo foi modificando-se com base em pesquisas científicas, as quais identificaram diferentes etiologias, graus de severidade e características específicas ou não usuais, deixando então de ser considerado um quadro único e passando a ser visto como uma síndrome. A tendência nas definições atuais de autismo é a de conceituá-lo como uma síndrome comportamental, de etiologias múltiplas, que compromete o processo do desenvolvimento infantil (Gillberg, 1990; Rutter, Taylor \& Hersov, 1996).

Atualmente, de acordo com o DSM-IV-TR (2002), os Transtornos Globais do Desenvolvimento caracterizam-se pelo comprometimento severo e invasivo em três áreas do desenvolvimento: habilidades de interação social recíproca, habilidades de comunicação, e presença de comportamentos, interesses e atividades estereotipadas. Nessa classificação estão incluídas cinco categorias diagnósticas: Transtorno Autista, o Transtorno de Rett, Transtorno Desintegrativo da Infância, Transtorno de Asperger e Transtorno Global do Desenvolvimento sem outra Especificação, sendo o autismo o transtorno prototípico desta categoria.

O comprometimento da interação social é caracterizado por alterações qualitativas das interações sociais recíprocas. Podem-se observar dificuldades na espontaneidade, imitação e jogos sociais, bem como uma inabilidade em desenvolver amizade com companheiros da mesma idade; comprometimento acentuado no uso de comportamentos verbais e nãoverbais, além da falta de reciprocidade social e emocional (Assumpção Jr., 1997; Bosa, 2002; Tanguay, 2000, Rutter \& cols., 1996). Quanto ao comprometimento das modalidades de comunicação, é relatado atraso na aquisição da fala, uso estereotipado e repetitivo da linguagem e uma inabilidade em iniciar e manter uma conversação. Acrescentam-se ainda outras características como a inversão pronominal (falar sobre si na terceira pessoa), a ecolalia imediata e a ecolalia diferida (Assumpção Jr., 1997; Baptista \& Bosa, 2002; Rutter \& cols., 1996).

O terceiro item da tríade refere-se aos padrões restritos e repetitivos de comportamentos, interesses e atividades. Estes podem ser manifestados através da adesão inflexível a rotinas e rituais específicos, não funcionais, e pela preocupação persistente com partes de objetos, em detrimento do objeto como um todo. Além destes sintomas, observa-se insistência na mesmice, bem como a manifestação de sofrimento e resistência frente a mudanças (DSM-IV-TR, 2002).

De acordo com o DSM-IV-TR (2002), os sintomas do autismo se fazem presentes antes dos 36 meses de idade. Não existe, em geral, um período de desenvolvimento inequivocamente normal, embora em 20\% dos casos os pais não tenham relatado comprometimentos durante os dois primeiros anos de vida da criança. Esse quadro é associado à deficiência mental em cerca de $75 \%$ dos casos, e poucos indivíduos apresentam QI accima de 80 (Facion, Marinho \& Rabelo, 2002). É de consenso que a maioria dos casos (mas não todos) que apresenta a tríade em grau severo, mostra os primeiros sintomas logo no início da vida. Portanto, é plausível supor que essas características exerçam um impacto no cotidiano das famílias e nas relações entre seus membros.

\section{O Impacto dos Transtornos Globais do Desenvolvimento na Família}

A família de indivíduos portadores de autismo se vê frente ao desafio de ajustar seus planos e 
expectativas quanto ao futuro, às limitações desta condição, além da necessidade de adaptar-se à intensa dedicação e prestação de cuidados das necessidades específicas do filho (Breslau \& Davis, 1986; Bristol \& Schopler, 1984; DeMyer, 1979; Gallagher, Beckman \& Cross, 1983; Gath, 1977; Gauderer, 1997; Harris, 1983; Richman, 1977). As características clínicas da síndrome afetam as condições físicas e mentais do indivíduo, aumentando a demanda por cuidados e, conseqüentemente, o nível de dependência de pais e/ou cuidadores. Essa situação pode constituir um estressor em potencial para familiares.

Vários estudos revelaram a existência de estresse agudo em famílias que possuem um membro com diagnóstico de autismo (Cutler \& Kozloff, 1987; Factor, Perry, \& Freeman, 1990; Perry, SarloMcGarvey \& Factor, 1992; Schopler \& Mesibov, 1984). O estudo de Bristol e Schopler (1983) complementa estes dados, demonstrando que os familiares de crianças com autismo apresentam padrões de estresse mais elevados do que famílias que possuem um filho com desenvolvimento típico ou com síndrome de Down, sugerindo que o estresse parece ser influenciado por características específicas do autismo e não apenas por um atraso do desenvolvimento. De fato, conforme apontado por estes autores, percebe-se uma inversão na ênfase histórica de pesquisas dos efeitos psicológicos do comportamento dos pais sobre a criança (Bettelheim, 1967; Eisenberg, 1956; Rank, 1949; Ruttenberg, 1971) para pesquisas dos efeitos psicológicos do comportamento das crianças sobre os pais.

Konstantareas e Homatidis (1989), realizaram uma breve revisão histórica sobre a questão do estresse em familiares de crianças com autismo. Segundo os autores, Holroyd, Brown, Wilker e Simmons (citados em Konstatareas \& Homatidis) foram os primeiros a investigar o tema, identificando níveis consideráveis de estresse nas 29 famílias pesquisadas. Subseqüentemente, um outro estudo (Holroyd \& McArthur, 1976) encontrou maiores níveis de estresse em pais de crianças com autismo, quando comparados a grupos de controle.

No final da década de 1970, DeMyer (1979) publicou seu trabalho acerca do estresse dos pais de crianças com autismo, os quais foram comparados a um grupo controle. Os resultados demonstraram maior presença de tensão física e psicológica em mães de crianças com autismo, culpa em $66 \%$ destas e incertezas quanto a habilidades maternais em $33 \%$. Neste estudo, os pais também se mostraram afetados, porém de modo indireto, isto é, reagiram ao sofrimento de suas esposas. A partir de então, iniciamse questionamentos a respeito das possíveis diferenças quanto ao estresse entre pais e mães de crianças com autismo.

Milgram e Atzil (1988) estudaram os diferentes papéis desempenhados por pais e mães de crianças com autismo em Tel-Aviv. Os resultados apontam que as mães tendem a apresentar maior risco de crise e estresse parental que os pais, devido à demanda dos cuidados com a criança. Conforme estes autores, existe uma expectativa social de que as mães tomem para si esses cuidados, assumindo-os mais do que os pais. Contudo, é mencionado o sentimento de desamparo destas pela falta de suporte dos maridos, manifestando o desejo de que eles assumam uma responsabilidade conjunta e espontânea acerca dos cuidados do filho. Estes achados são corroborados pelo estudo de Konstantareas e Homatidis (1989), em que foi examinado o nível de suporte conjugal disponível a mães e pais de crianças com autismo. Através de entrevistas semi-estruturadas, foram agrupados hierarquicamente os principais tipos de suporte desejados pelas mães, em relação aos seus cônjuges, sendo estes: 1) oportunizar maior alívio materno quanto aos cuidados da criança com autismo; 2) assumir maior responsabilidade disciplinar para com o filho; 3) obter ajuda paterna de forma espontânea, sem a necessidade de contínuos pedidos de ajuda.

Milgram e Atzil (1988), também descrevem o papel dos pais, que em contraposição às expectativas das mães, consideram justa sua menor participação nos cuidados gerais da criança, devido ao peso de suas responsabilidades financeiras e ocupacionais já desempenhadas junto à família. Porém, outros estudos revelam que estas responsabilidades tendem a acarretar tensão emocional e financeira significativas, conseqüentemente dificultando a contribuição destes para com os cuidados da criança (Bristol \& Gallagher, 1986; DeMyer, 1979; Milgram \& Atzil, 1988; PriceBonham \& Addison, 1978).

No início da década de 80, Bristol e Schopler (1983) relataram os achados de uma pesquisa não publicada de Bristol (1979, citado em Bristol \& Schopler), em que reafirmam que o grau de dependência, incapacidades cognitivas e as dificuldades nas características de comportamento da criança com autismo resultavam em maior estresse parental. Conforme Konstantareas e Homatidis (1989), tais dados conduzem mais a tentativas explicativas do que a informações conclusivas sobre a questão do estresse em familiares de sujeitos com autismo.

No início dos anos 1990, levantou-se a hipótese de que pudesse existir um padrão particular de áreas que influenciasse o estresse em pais de crianças com autismo. Koegel e colaboradores (1992) buscaram explorar melhor este tema. Para avaliar a consistência destes padrões de estresse, 50 famílias oriundas de várias regiões geográficas dos Estados Unidos e Alemanha preencheram o QRS - Questionnaire on Resources and Stress (Holroyd, 1974), levando em conta também o nível de funcionamento (QI) e a idade dos indivíduos (entre 3 e 31 anos). A correlação para mães de crianças com autismo, em cada uma das três localidades geográficas, mostrou-se bastante alta e estatisticamente significativa $(\mathrm{p}<0,001)$. O coeficiente de correlação entre o grupo da Califórnia (Santa Bárbara e São Diego) e o grupo da Alemanha foi de 0,931. Para o grupo da Califórnia versus o grupo Apalache (Kentucky, West Virginia e Ohio) a correlação foi de 0,957 . Por último, entre o grupo da Alemanha e o grupo Apalache a correlação foi de 0,886 . Os resultados sugerem a existência de um perfil 
característico de estresse para pais de crianças com autismo, mostrando uma constância relativa entre famílias de diferentes localidades geográficas. Dentre os itens avaliados, o que mostrou maior consistência quanto ao estresse em pais e mães foram as preocupações relativas ao bem-estar de seus filhos, quando os mesmos não puderem mais prover seus cuidados. Estas preocupações subdividem-se em: 1) preocupação com o futuro de seu filho; 2) dificuldades cognitivas e habilidades de funcionamento independente da criança; e 3) aceitação em sua comunidade.

Consistente com os achados de outros pesquisadores (Bristol \& Schopler, 1983; Holroyd \& McArthur, 1976), estes resultados sugerem que o convívio e os cuidados continuados prestados a crianças com autismo se mostra como um poderoso estressor, que age sobre a vida destas famílias de modo muito característico.

A identificação de fontes específicas de estresse em familiares de pessoas com Transtornos Globais do Desenvolvimento também foi foco de interesse de Perry e colaboradores (1992). Os autores encontraram níveis de estresse mais elevados em familiares de crianças portadoras da síndrome de Rett quando comparados a grupos controle. Os resultados mostraram que os pais de meninas com síndrome de Rett experienciam maior estresse em relação aos seus sentimentos de apego para com suas filhas, isolamento social e problemas de saúde, do que o grupo controle. A justificativa dos autores é de que os níveis de estresse mais elevados, nesta população em particular, se devem a vários fatores: a) a aparência saudável da criança quando bebê cria expectativas nos pais, que são posteriormente destruídas; b) os filhos podem apresentar incapacidades graves, necessitando de cuidados intensos durante toda a vida; c) as poucas expectativas quanto a melhorias de tratamento.

Embora grande parte da literatura mostre evidências de maiores níveis de estresse em familiares de crianças com transtornos no desenvolvimento (Koegel \& cols., 1992; Perry \& cols., 1992), outras pesquisas abordam este assunto sob outra ótica. Alguns estudos (Konstantareas, Homatidis \& Plowright, 1992; Silva \& Dessen, 2003) indicam que as dificuldades das crianças com algum transtorno do desenvolvimento podem ser consideradas como um estressor apenas em potencial, podendo estes pais sofrer ou não os efeitos de um estresse real. Para estes autores, o impacto das dificuldades próprias da síndrome sobre os pais vai depender de uma complexa interação entre a severidade das características próprias da criança e as de personalidade dos pais, bem como a disponibilidade de recursos comunitários e sociais. De fato, Gomes e Bosa (2002), ao investigarem o impacto do autismo em irmãos de portadores dessa condição, não encontraram evidência de estresse nesta população. Na verdade, o nível de estresse estava mais associado à qualidade das relações familiares (Ex.: conflito e punição), independentes de ter ou não um irmão com autismo.

De acordo com Bristol e Schopler (1983), na área da sociologia têm-se procurado desenvolver modelos de avaliação que identifiquem fatores predisponentes a crises familiares face ao estresse. Hill (1949) já havia chamado a atenção para a necessidade de um modelo compreensivo de estresse familiar, que levasse em conta a avaliação dos recursos familiares disponíveis. De forma similar, Konstantareas e colaboradores (1992) sugerem uma avaliação sistemática do estresse percebido, por exemplo, enfocando a forma como estes pais encontram-se frente à necessidade de lidar com a alta demanda de cuidados solicitados por seus filhos, às reações negativas da comunidade, aos limitados recursos de apoio que podem contar ou sentimentos diante de possíveis renúncias quanto à carreira profissional.

Um estudo anterior, destas mesmas autoras (Konstantareas \& Homatidis, 1989), buscou investigar o estresse familiar percebido, a partir de três aspectos: as características da criança, características de personalidade dos pais e recursos de coping. Os objetivos foram: 1) identificar a forma como pais e mães avaliam a presença e o grau de expressão de 14 sintomas-chave de autismo em seu filho; 2) avaliar o quão sintomático os pais percebem seu filho, clareando a relação entre severidade do sintoma e estresse; 3) avaliar o estresse em função de cada sintoma para determinar possíveis diferenças quanto ao impacto destes em pais e mães; 4) avaliar como a severidade do sintoma e o estresse concomitante se relacionam com algumas características da criança; e 5) examinar os efeitos de outras características familiares (nível socioeconômico, tamanho da família, suporte social) sobre os níveis de estresse.

Para tanto, 44 famílias, que tinham filhos com autismo, participaram do estudo, sendo que a faixa de idade dos pais variava de 23 a 52 anos. Foram utilizadas uma escala e uma entrevista semiestruturada para avaliar o grau de suporte social e dificuldades experienciadas ao lidar com agentes sociais e comunitários. Quanto aos resultados, a percepção dos sintomas mostrou-se influenciada pela idade da criança, sendo as crianças mais novas (até 6 anos) vistas como menos sintomáticas pelos pais em comparação à percepção dos clínicos. O estudo também identificou alto grau de concordância interparental quanto à avaliação da severidade dos sintomas: 1) pobreza na comunicação; 2) discrepâncias nas habilidades cognitivas; 3) comprometimento no relacionamento interpessoal.

No caso do estresse parental devido aos sintomas da criança, a pontuação foi equivalente entre pais e mães, porém enquanto o melhor preditor para o estresse paterno foi os comportamentos autoabusivos (bater-se, arranhar-se, beliscar-se ou morder-se), para as mães foi a hiperirritabilidade (grau de inquietude e comportamentos disfuncionais) e a idade mais avançada da criança (6 a 12 anos).

Estudos têm apresentado dados a respeito da natureza dos eventos causadores de estresse e das características próprias da criança com autismo, que exercem um impacto sobre os familiares, considerando como parte integrante deste processo, as formas utilizadas pelos familiares para lidar com o estresse (coping) (Schopler \& Mesibov, 1983; 
Konstantareas \& cols., 1992). As emoções e a excitação fisiológica criada por situações estressantes são altamente desconfortáveis, motivando o indivíduo a fazer algo para seu alívio (Atkinsons, Atkinsons, Smith \& Bem, 1995; Lazarus \& Folkman, 1984). Lazarus e Folkman identificaram duas formas principais de coping. A primeira inclui estratégias de definição do problema, geração de soluções alternativas, comparação em termos de custos e benefícios, seleção e implementação da alternativa escolhida (focalizada no problema). Já a segunda, focalizada na emoção, objetiva desenvolver ações para controlar o estado emocional, bem como impedir que as emoções "negativas" afetem o engajamento em ações para a solução de seus problemas.

Tunali e Power (1993) abordaram a relação entre estresse e coping em pais de crianças com transtornos do desenvolvimento. Conforme sua análise, quando um indivíduo se encontra em uma situação inevitável em que uma necessidade humana básica está ameaçada, esta exposição ao estresse pode "redefinir o preenchimento destas necessidades, desenvolvendo meios alternativos para alcançá-lo" (p. 950). Baseado neste modelo, em um estudo posterior (Tunali \& Power, 2002), os autores compararam as respostas de mães de crianças com e sem autismo a respeito de uma série de tópicos envolvendo o seu cotidiano, em uma entrevista. Os resultados não revelaram diferenças entre os grupos quanto a itens como ajuste psicológico, satisfação de vida ou satisfação conjugal. Contudo, os autores encontraram uma correlação positiva entre determinadas estratégias de coping e satisfação de vida, em mães de filhos com autismo. Por exemplo, as mães que apresentaram maior satisfação de vida, foram aquelas que tendiam a depositar maior ênfase na importância de "ser uma boa mãe" do que na carreira profissional; a passar a maior parte de seu tempo de lazer com familiares; a depositar menor ênfase no lazer individual e na opinião ou aprovação alheias a respeito dos comportamentos de seus filhos; maior ênfase nos papéis e suporte parentais do que na intimidade conjugal e lazer compartilhado; maior tolerância à ambigüidade, tanto em relação a seus filhos quanto no geral. Essa correlação foi negativa para mães de crianças sem autismo.

Os estudos apresentados mostram que os pais ou cuidadores de um indivíduo portador de autismo lidam diariamente com diversos estressores. Esta condição afeta todos os membros da família, causando estresse direta (Ex: pobreza na interação social) ou indiretamente (Ex: impacto financeiro).

A análise da literatura revisada revela contradições entre as pesquisas quanto às características comportamentais da criança com autismo que mais contribuem para o estresse em familiares. O estudo de Konstantareas e Homatidis (1989), por exemplo, comparou dados de famílias de crianças com e sem autismo a fim de mapear as principais fontes do estresse familiar. $\mathrm{O}$ melhor preditor para o estresse paterno foi os comportamentos autoabusivos e para o materno foi a hiperirritabilidade. É interessante notar que ambos os comportamentos citados não são típicos ou exclusivos do autismo, podendo ser encontrados em outras condições (Ex: deficiência mental). Logo, o estresse produzido por estes fatores pode estar presente em outras famílias que não somente a de crianças com autismo.

Por outro lado, no estudo de Bebko, Konstantareas e Springer (1987), a maior fonte de estresse parental foram os déficits na comunicação (linguagem verbal expressiva) e cognitivos (discrepância entre diferentes áreas). Considerando que as dificuldades na comunicação afetam o relacionamento interpessoal, e constituem critérios-chave para o diagnóstico de autismo, pode-se compreender estes resultados como sendo mais específicos aos casos de autismo.

De forma similar, a pesquisa realizada por Koegel e colaboradores (1992) também identificou as dificuldades verbais e cognitivas das crianças com autismo como os sintomas mais relacionados ao estresse em familiares. Porém, analisando a metodologia desta pesquisa em pormenor, nota-se que não foi levado em conta o apoio social disponível a estas famílias nem a severidade dos sintomas presentes na criança. Sabe-se que estes dois fatores exercem influência importante quanto ao mapeamento do estresse parental (Factor \& cols., 1990; Gill \& Harris, 1991; Konstantareas \& cols., 1992).

Além disso, os autores examinaram a associação entre idade da criança e o estresse familiar e concluíram não haver relação significativa entre estes fatores. Outros estudos contrariaram estes achados (Bristol \& Schopler, 1983; DeMyer \& Goldberg, 1983) encontrando que a idade mais avançada da criança (adolescentes) tende a ser preditor de estresse familiar. Uma possível explicação para isso é que, enquanto as crianças são pequenas, os pais ainda não têm plena consciência do significado e da extensão de seus "sintomas", gerando menor preocupação. Já em outros estudos (Bebko \& cols., 1987; Schopler \& Mesibov, 1983) os pais consideraram os sintomas das crianças mais velhas (adolescentes) como menos severos, contribuindo para as contradições quanto aos achados.

Outro ponto ainda divergente apresentado nas pesquisas se refere às diferenças de estresse percebido por pais e mães. Considera-se que o papel social esperado e a maior responsabilidade sobre os cuidados por parte das mães, os quais são intensos e prolongados, as tornem alvo de maior estresse. Outros estudos que diferenciam o estresse em pais e mães consideram que o estresse sofrido pelo pai é qualitativamente diferente do das mães, não implicando em maior ou menor estresse (Bristol \& Gallagher, 1986; DeMyer \& Goldberg, 1983).

Já Factor e colaboradores (1990) avaliaram separadamente 36 pais e mães de crianças com e sem autismo, revelando não haver diferenças estatisticamente significativas para níveis de estresse entre ambos. Em contraponto, grande parte das pesquisas considera as mães como mais afetadas pelo estresse que os pais (Bristol \& Gallagher, 1986; DeMyer, 1979; Konstantareas \& Homatidis, 1989; Milgram \& Atzil, 1988). Os resultados parecem 
apontar para uma sobrecarga maior nas mulheres, pelo menos em termos de cuidado direto da criança. Esse fator parece estar associado ao estresse percebido e às consequientes dificuldades experienciadas quanto a habilidades (ou não) no manejo com os filhos portadores de TGD.

Além dos achados controversos apresentados na literatura, percebe-se o fato de que a maior parte das pesquisas utiliza-se predominantemente de escalas fechadas como instrumento, obtendo dados relevantes, porém limitados. É importante a utilização de instrumentos menos estruturados, como entrevistas, além de escalas, a fim de se ampliar a compreensão do fenômeno, a partir da exploração do ponto de vista do participante. Por último, cabe salientar também a escassez de pesquisas que abordam esta temática na população brasileira. Alguns dos poucos estudos encontrados investigam a questão familiar na área do autismo sob o enfoque da psicanálise (Prado, 1993, 1999) e/ou da teoria sistêmica (Sprovieri \& Assumpção Jr., 2001). Estes estudos relacionam os déficits típicos do autismo ao funcionamento familiar, concluindo que a família do portador de TGD é dificultadora da saúde emocional de seus membros e, conseqüentemente, da adaptação familiar. $\mathrm{O}$ modelo utilizado para a compreensão é um modelo de déficit, ou seja, a adaptação da família é relacionada ao grau e intensidade dos comprometimentos dos TGD. As conclusões deste modelo de pesquisa parecem conduzir à idéia de que o estresse familiar é inerente à presença de um membro com TGD na família, sem preocupações em apontar possíveis saídas, formas de minimização do sofrimento presente ou inter-relações entre os fatores os quais podem facilitar ou, ao contrário, limitar o desenvolvimento psicossocial do grupo familiar.

Em contraponto a esta perspectiva, alguns estudos lançam mão de outros modelos, chamados de "modelos de recurso" (Tunali \& Power, 2002; Beresford, 1994; Factor \& cols., 1990) nos quais a ênfase passa a ser dada aos recursos da família que podem ser utilizados para favorecer sua adaptação a uma condição que afeta a saúde de forma crônica. Estes estudos não se limitam a identificar fatores de risco para a adaptação da família, mas a investigar de que forma os diferentes recursos combinam-se na promoção da saúde (Ex.: estratégias de coping utilizadas pelos pais, traços de personalidade, qualidade do sistema de saúde etc). Recentemente, tem havido esforços de se compreender o autismo a partir de um conceito mais amplo de saúde, articulando-se noções da psicologia da saúde e do desenvolvimento (Gomes \& Bosa, 2002).

O modelo proposto por Bradford (1997) abarca tanto os modelos de déficit quanto os modelos de recurso, integrando-os num "metamodelo". A partir da perspectiva do autor, o modelo biopsicossocial permite ampliar a investigação do fenômeno, integrando concepções oriundas da teoria sistêmica (Ex.: padrões de comunicação e interações familiares), da psicologia da saúde (Ex.: desafios específicos da doença, aspectos crônicos e qualidade dos sistemas de saúde) e da psicologia do desenvolvimento e personalidade (Ex.: redes de apoio e características de personalidade dos membros da família), com ênfase em teorias cognitivas (Ex.: coping e crenças sobre saúde).

Apesar deste modelo ter sido desenvolvido no âmbito da doença orgânica crônica, portanto no campo da psicologia da saúde, sua natureza multifacetada ao compreender o impacto de uma condição crônica na família tem sido também de grande utilidade na compreensão dos transtornos globais do desenvolvimento. Isso porque essa condição implica comprometimentos (Ex.: interação social) em etapas precoces do desenvolvimento, que tendem a perdurar ao longo do ciclo vital da família. Portanto, trata-se de um processo crônico. Ademais, esse modelo não desconsidera as discussões em torno das dimensões biológicas de uma determinada condição. Na questão do autismo, pode-se focalizar, por exemplo, fatores genéticos, associação com epilepsia etc. sem perder de vista as possíveis interações com o ambiente, ao explicar as variações individuais na expressão comportamental da síndrome. Em outras palavras, a questão crucial desloca-se da contraprodutiva discussão em torno da divisão "doença mental x neuropsicológica" para uma concepção global de saúde.

$\mathrm{O}$ modelo de adaptação à doença crônica de Bradford (1997), postula que a doença crônica é uma condição específica que requer cuidados especiais. Apesar de não haver um consenso sobre uma definição acerca do que se considera como "crônico", há uma tendência em considerar esse termo como se referindo a períodos de agravamento das condições de saúde relativamente longos ou recorrentes no tempo (Bradford). Um dos diferenciais dessa abordagem em relação a outros modelos é a noção de que a presença de psicopatologia familiar não é vista como uma conseqüência direta e inquestionável da ocorrência de doenças crônicas na família, mas como um processo que depende, entre outros aspectos, da forma como a família maneja os estressores. $\mathrm{O}$ autor se contrapõe à idéia de que o ajuste, ou a adaptação familiar equivale à ausência de patologia. A ênfase maior é dada à forma como a criança e sua família se comportam frente aos vários desafios inerentes a esta situação, baseando-se numa perspectiva sistêmica para o entendimento do fenômeno. Os fatores que permeiam a adaptação familiar a enfermidades crônicas são tidos como potencialmente estressantes, acometendo mais ou menos a família, conforme a adaptação às exigências de mudanças externas ou internas. De acordo com Minuchin (1990), esta adaptação será responsável pelo fornecimento de um esquema de referência, caracterizando o grupo familiar. A prioridade é dada à totalidade do sistema familiar, através da dinâmica de suas características próprias (Schaffer, 1996).

A totalidade do sistema familiar pode ser dividida em subsistemas internos, caracterizados pelo relacionamento entre seus membros, como por exemplo, as relações filho-filho, pai-filho, mãe-filho, pai-mãe, etc. (Minuchin, 1990). Cada membro pertence a diversos subsistemas que caracterizam 
padrões próprios de comportamentos. Assim, as fronteiras entre cada subsistema são definidas pela determinação de regras que definem as formas de participação de cada membro.

Conforme Morgan (1988), os relacionamentos entre os diferentes componentes da família são caracterizados pelas interações recíprocas e bidirecionais. Os padrões de influência dentro do sistema ocorrem de forma circular, estabelecendo uma relação de mútua interdependência entre seus membros. Desta forma, as condições presentes em um dos membros (Ex: estresse) tenderá a repercutir nos demais subsistemas (Ex.: estresse familiar), afetando a família como um todo. Considerando a reciprocidade entre os componentes, considera-se que os padrões de interação dos pais com o filho afetam ao mesmo tempo em que são afetados pela relação do filho com estes pais (Schaffer, 1996).

Considerando o estresse como um destes desafios, Minuchin (1990) coloca que o sistema familiar pode ser confrontado com quatro principais fontes de estresse: 1) contato estressante de um membro com forças extrafamiliares; 2) contato estressante de toda a família com forças extrafamiliares; 3) estresse em pontos de transição na família; e 4) estresse em torno de problemas idiossincráticos.

O primeiro fator de estresse diz respeito ao contato estressante de um membro com forças extrafamiliares, como por exemplo, a perda do emprego, em que todos os componentes da família sentem a necessidade de se ajustar às novas circunstâncias enfrentadas por um de seus membros. O segundo fator de estresse citado envolve todos os membros do sistema familiar. $\mathrm{O}$ contato estressante de toda a família com forças extrafamiliares (Ex: mudança de cidade), conduz a uma necessidade de ajuste às novas mudanças enfrentadas por todos que pertencem ao sistema.

O estresse em pontos de transição da família é caracterizado por mudanças inerentes ao ciclo de desenvolvimento familiar, por exemplo, quando nasce uma nova criança ou o ingresso de um de seus membros na fase da adolescência. Neste período várias regras familiares sofrem a necessidade de reajuste, desencadeando conflitos que poderão ser resolvidos através de negociações de transição, acarretando crescimento a todos os seus membros.

Por fim, o estresse em torno de problemas idiossincráticos se refere às dificuldades surgidas em detrimento do tempo. Por exemplo, a família que se encontra ajustada em determinado momento às demandas da doença crônica em um de seus membros pode vir a ter que renegociar futuros desafios face às novas demandas próprias do desenvolvimento humano em suas fases subseqüentes. À medida que a criança cresce, novos problemas surgem, trazendo a necessidade de novos ajustes. Com isso, pode-se dizer que a "adaptação" familiar não é linear e progressiva, ocorrendo, ao contrário, em "picos".

Dentro do modelo de adaptação à doença crônica, Bradford (1997) chama a atenção para a interação entre os fatores relevantes ao contexto, os quais estão representados na Figura 1: Crenças sobre saúde, sistemas de saúde, padrões de interação familiar e padrões de comunicação intra (entre os membros da família) e extrafamiliar (entre a família e o sistema de saúde). Estes diversos fatores são compreendidos a partir de seu potencial para interagirem mutuamente, possibilitando inúmeras associações entre fatores contributivos, ampliando a magnitude da investigação.

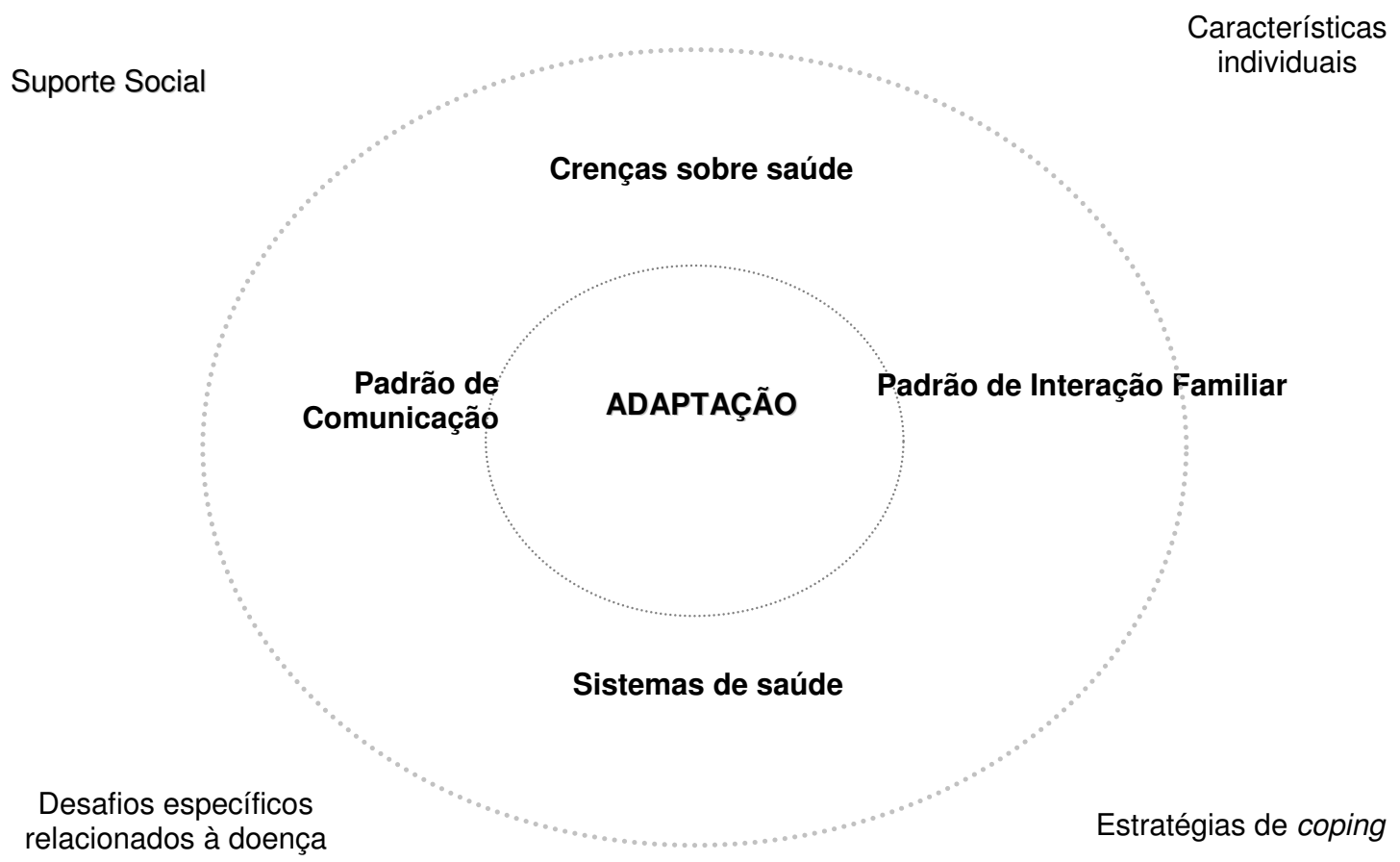

Figura 1 - Modelo de Bradeford (1997). 
Para esse autor, a identificação das crenças subjacentes é um fator relevante, pois há evidências de que esse fator é um importante preditor da adaptação familiar e do próprio paciente e independe da gravidade ou grau de incapacitação de uma determinada enfermidade. Posição similar é adotada por McDaniel, Hep-worth e Doherty (1994) ao afirmarem que algumas famílias podem ser fatalistas acerca das suas crenças sobre uma determinada enfermidade (Ex.: acreditar que a mesma é parte de um plano divino maior e está fora do seu controle ou o resultado de negligência). Valores familiares e crenças religiosas também podem influenciar o processo decisório sobre o tratamento.

Beresford (1994) complementa que as crenças religiosas de pais de filhos com dificuldades não são estratégias de coping importantes apenas no momento do diagnóstico e início dos sintomas, mas um recurso potencial relevante durante todo o processo de prestação de cuidados à criança. Este autor relata que crenças religiosas podem oferecer uma maneira de interpretar ou redefinir o significado das dificuldades do filho. Algumas crenças permitem aos pais acreditar terem sido especialmente selecionados para lidar com seu filho com problemas, ou ainda, que estes recebem "forças" para executar esta tarefa (Beresford, 1994).

A pesquisa desenvolvida por Nihira, Meyers e Mink (1980), a respeito do papel das crenças religiosas na adaptação familiar de pais de crianças com deficiência mental encontrou uma associação positiva entre a religiosidade e o modo eficaz com que familiares lidam com os problemas de seus filhos. Por outro lado, Beresford (1994) relata que as crenças também podem desenvolver vulnerabilidades quando estas conduzem os familiares a culparem-se pela condição da criança.

\section{CONSIDERAÇÕES FINAIS}

O impacto dos TGDs na família tem sido foco de investigações há quase três décadas, revelando intenso interesse científico pelo tema. As pesquisas que inicialmente enfatizavam os efeitos dos pais sobre a criança, passaram a estudar os efeitos da criança com TGD sobre os pais. De acordo com esta mudança, estudos atuais têm considerado aspectos como a natureza crônica da síndrome e o conseqüente acometimento dos pais pelo estresse decorrente da prestação de cuidados em longo prazo, como fatores importantes para a compreensão do fenômeno.

Apesar dos avanços já obtidos nesta área, resultados controversos identificam diferentes fatores associados ao impacto dos TGDs. Estas inconsistências são, possivelmente, reflexos de falhas metodológicas ou divergências teóricas entre pesquisadores. Ao mesmo tempo em que essas diferenças nos achados dificultam a compreensão do impacto dos Transtornos Globais do Desenvolvimento na família, mostram a importância em desenvolver novos modelos de investigação do fenômeno, que ampliem a magnitude de fatores envolvidos neste processo. Nesse sentido, o metamodelo biopsicossocial de Bradford (1997) apresenta-se como uma alternativa, ao buscar conjugar, principalmente, noções da teoria sistêmica e cognitiva. A transposição do modelo, originalmente desenvolvido na área da Psicologia da Saúde, para a área da psicopatologia do desenvolvimento pode ser profícua, na medida em que não separa aspectos orgânicos dos mentais. Em outras palavras, é possível trabalhar com uma noção integrada de saúde, ao invés de se separar a "mente" do "corpo". Particularmente importante nesse modelo é a preocupação quanto à compreensão de como diversos fatores operam e interatuam em condições adversas prolongadas, atingindo a família em diferentes etapas do seu ciclo vital. Entretanto, cabe salientar que mais evidências empíricas necessitam ser produzidas em favor do modelo para que as noções aqui discutidas tornem-se mais conclusivas.

\section{REFERÊNCIAS}

Assumpção Jr., J. F. B. (1997). Transtornos invasivos do desenvolvimento infantil. São Paulo: Lemos.

Atkinsons, R. L.; Atkinsons, R. C.; Smith, E. E. \& Bem, D. J. (1995). Introdução à psicologia. Porto Alegre: Artes Médicas.

Baptista, C. \& Bosa, C. (2002). Autismo e educação: Reflexões e propostas de intervenção. Porto Alegre: Artmed.

Bebko, J. M.; Konstantareas, M. M. \& Springer, J. (1987). Parent and professional evaluations of family stress associated with characteristics of autism. Journal of Autism and Developmental Disorders, 17, 565-576.

Beresford, B. A. (1994). Resources and strategies: How parents cope with the care of a disable child. Journal of Child Psychology and Psychiatry, 35, 171-209.

Bettelheim, B. (1967). The empty fortress: Infantile autism and the birth of the self. New York: Free Press.

Bosa, C. (2002). Atuais interpretações para antigas observações. Em C. Baptista \& C. Bosa (Orgs.), Autismo e educação. Reflexões e propostas de intervenção (pp. 21-40). Porto Alegre: Artmed.

Bradford, R. (1997). Children, families and chronic disease: Psychological models and methods of care. London: Routledge.

Breslau, N. \& Davis, G. C. (1986). Chronic stress and major depression. Archives of General Psychiatry, 43, 309-314.

Bristol, M. M. \& Gallagher, J. J. (1986). Research on fathers of young handicapped children: Evolution, review and some future directions. Em J. Gallagher \& P. M. Vietze (Orgs.), Families of handicapped persons: Research, programs and policy issues (pp. 81-100). Baltimore, MD: Brookes.

Bristol, M. M. \& Schopler, E. (1983). Coping and stress in families of autistic adolescents. Em E. Schopler \& G. B. Mesibov (Orgs.), Autism in adolescents and adults (pp. 251276). New York: Plenum.

Bristol, M. M. \& Schopler, E. (1984). A developmental perspective on stress and coping in families of autistic children. Em J. Blancher (Org.), Severely handicapped young children and their families (pp. 231-251). Orlando, Florida: Academic Press.

Cutler, B. C. \& Kozloff, M. A. (1987). Living with autism: Effects on families and families needs. Em D. J. Cohen \& A. M. Donnellan (Orgs.), Handbook of autism and pervasive developmental disorders (pp. 513-527). New York: Wiley.

DeMyer, M. K. (1979). Parents and children in autism. New York: Wiley.

DeMyer, M. K. \& Goldberg, P. (1983). Family needs of the autistic adolescent. Em E. Schopler \& G. B. Mesibov (Orgs.). 
Autism in adolescents and adults (pp. 225-250). New York: Plenum Press.

Silva, N. L. \& Dessen, M. A. (2003). Crianças com Síndrome de Down e suas interações familiares. Psicologia: Reflexão $e$ Crítica, 16, 503-514.

Eisenberg, L. (1956). The autistic child in adolescence. American Journal of Psychiatry, 112, 607-612.

Facion, J. R.; Marinho, V. \& Rabelo, L. (2002). Transtorno autista. Em J. R Facion (Org.), Transtornos invasivos do desenvolvimento associados a graves problemas do comportamento: Reflexões sobre um modelo integrativo (pp. 23-38). Brasília: CORDE.

Factor, D.C.; Perry, A. \& Freeman, N. (1990). Brief report: Stress, social support, and respite care in families with autistic children. Journal of Autism and Developmental Disorders, 20 , 139-146.

Gallagher, J. J.; Beckman, P. \& Cross, A. H. (1983). Families of handicapped children: Sources of stress and its amelioration. Exceptional Children, 50, 10-19.

Gath, A. (1977). The impact of an abnormal child upon the parents. British Journal of Psychiatry, 130, 405-410.

Gauderer, C. (1997). Autismo e outros atrasos no desenvolvimento. Rio de Janeiro: Revinter.

Gill, M. J. \& Harris, S. L. (1991). Hardiness and social support as predictors of psychological discomfort in mothers of children with autism. Journal of Autism and Developmental Disorders, $21,407-416$

Gillberg, C. (1990). Autism and pervasive developmental disorders. Journal of Child Psychology and Psychiatry, 31, 99-119.

Gomes, V. \& Bosa, C. (2002). Estresse e relações familiares na perspectiva de irmãos de indivíduos com e sem Transtornos Globais do Desenvolvimento. Dissertação de Mestrado não publicada, Curso de Pós-Graduação em Psicologia do Desenvolvimento, Universidade Federal do Rio Grande do Sul. Porto Alegre, RS.

Harris, S. L. (1983). Families of developmentally disabled children: A guide to behavioral intervention. New York: Pergamon.

Hill, R. (1949). Families under stress. New York: Harper.

Holroyd, J. (1974). The questionnaire on resources and stress: An instrument to measure family response to a handicapped member. Journal of Community Psychology, 2, 92-94.

Holroyd, J. \& McArthur, D. (1976). Mental retardation and stress on the parents: A contrast between Down's syndrome and childhood autism. American Journal of Mental Deficiency, 80, 431-438.

Kanner, L. (1943). Autistic disturbances of affective contact. Nervous Child, 2, 217-250.

Kanner, L. (1944). Early infantile autism. Journal of Pediatrics, $25,211-217$

Koegel, R. L.; Schreibman, L.; Loos, L. M.; Dilrich-Wilhelm, H.; Dunlap, G.; Robbins, R. \& Plienis, A. J. (1992). Consistent stress profiles in mothers of children with autism. Journal of Autism and Developmental Disorders, 22, 205-215.

Konstantareas, M. M. \& Homatidis, S. (1989). Assessing child symptom severity and stress in parents of autistic children. Journal of Psychology and Psychiatry, 30, 459-470.

Konstantareas, M.; Homatidis, S. \& Plowright, C. M. (1992). Assessing resources and stress in parents of severely dysfunctional children trough the Clarke modification of Holroyd's Questionnaire on Resources and Stress. Journal of Autism and Developmental Disorders, 22, 217-234.

Lazarus, S. \& Folkman, S. (1984). Stress, appraisal and coping. New York: Springer.

Manual Diagnóstico e Estatístico de Transtornos Mentais (DSMIV-TR, 2002). Porto Alegre: Artes Médicas.

McDaniel, S. H.; Hepworth, J. \& Doherty, W. (1994). Terapia familiar médica. Porto Alegre: Artmed.
Milgram, A. \& Atzil, M. (1988). Parenting stress in raising autistic children. Journal of Autism and Developmental Disorders, 18, 415-424.

Minuchin, S. (1990). Famílias, funcionamento e tratamento. Porto Alegre: Artes Médicas.

Morgan, S. (1988). The autistic child and family functioning: A developmental-family systems perspective. Journal of Autism and Developmental Disorders, 18, 263-280.

Nihira, K.; Meyers, C. E. \& Mink, I. T. (1980). Home environment, family adjustment and the development of mentally retarded children. Applied Research in Mental Retardation, 1, 5-24.

Perry, A.; Sarlo-McGarvey, N. \& Factor, D. (1992). Stress and family functioning in parents of girls with Rett syndrome. Journal of Autism and Developmental Disorders, 22, 235-248.

Prado, M. C. (1993). A psicanálise da família autista. Informação Psiquiátrica, 12, 102-105.

Prado, M. C. (1999). Entrando em contato com o mundo da família autista. Informação Psiquiátrica, 18, 3-13.

Price-Bonham, S. \& Addisson, S. (1978). Families and mentally retarded children. Family Coordinator, 27, 221-230.

Rank, B. (1949). Adaptation of the psychoanalytic techniques for the treatment of young children and atypical development. American Journal of Orthopsychiatry, 19, 130-139.

Richman, N. (1977). Behavior problems in preschool children: Family and social factors. British Journal of Psychiatry, 131 525-527.

Ruttemberg, B. A. (1971). A psychoanalytic understanding of infantile autism and its treatment. Em D. W. Churchill; G. D. Alpern \& M. K. DeMyer (Orgs.), Infantile autism (pp. 76-90). Springfield, IL: Charles C. Thomas.

Rutter, M.; Taylor, E. \& Hersov, L. (1996). Child and adolescent psychiatry: Modern approaches. Oxford: Blackwell Science.

Schaffer, R. (1996). Social development. Oxford: Blackwell.

Schopler, E. \& Mesibov, G. B. (1983). Autism in adolescents and adults. New York: Plenum.

Schopler, E. \& Mesibov, G. B. (1984). The effect of autism on the family. New York: Plenum.

Sprovieri, M. H. \& Assumpção Jr., J. F. B. (2001). Dinâmica familiar de crianças autistas. Arquivos de Neuropsiquiatria, $59,230-237$.

Tanguay, P. E. (2000). Pervasive developmental disorder: A 10year review. Journal of Academy of Child and Adolescent Psychiatry, 39, 1079-1095.

Tunali, B. \& Power, T. (1993). Creating satisfaction: A psychological perspective on stress and coping in families of handicapped children. Journal of Child Psychology and Psychiatry, 34, 945-957.

Tunali, B. \& Power, T. (2002). Coping by redefinition: Cognitive appraisals in mothers of children with autism and children without autism. Journal of Autism and Developmental Disorders, 32, 25-34.

Recebido: 10.09 .2003

Revisado: 22.10 .2003

Aceito: 03.12.2003 


\section{Sobre os autores:}

Carlo Schmidt: Psicólogo, Mestrando em Psicologia do Desenvolvimento pelo Instituto de Psicologia da Universidade Federal do Rio Grande do Sul - UFRGS - E-mail: carlopsi@ hotmail.com

Cleonice Bosa: Psicóloga, PhD em Psicologia pela Universidade de Londres, Professora do Programa de Pós-Graduação em Psicologia do Desenvolvimento do Instituto de Psicologia da Universidade Federal do Rio Grande do Sul - UFRGS - E-mail: cleobosa@uol.com.br 